\title{
Measurement of high- $Q^{2}$ neutral current deep inelastic $e^{+} p$ scattering cross-sections with a longitudinally polarised positron beam at HERA
}

\author{
lan BROCK* \\ (Universität Bonn $(D E)$ ) \\ E-mail: ian.brock@cern.ch
}

on behalf of the ZEUS Collaboration

\begin{abstract}
Cross-sections for neutral current deep inelastic scattering in $e^{+} p$ collisions with a longitudinally polarised positron beam have been measured using the ZEUS detector at HERA. The singledifferential cross-sections $\mathrm{d} \sigma / \mathrm{d} Q^{2}, \mathrm{~d} \sigma / \mathrm{d} x$ and $\mathrm{d} \sigma / \mathrm{d} y$ and the double-differential cross-sections in $Q^{2}$ and $x$ are measured in the kinematic region $Q^{2}>185 \mathrm{GeV}^{2}$ for both positively and negatively polarised electron beams and for each polarisation state separately. The measurements are based on an integrated luminosity of $136 \mathrm{pb}^{-1}$ taken in 2006 and 2007 at a centre-of-mass energy of $318 \mathrm{GeV}$. The structure functions $x F_{3}$ and $x F_{3}^{\gamma Z}$ are determined by combining the $e^{+} p$ results presented in this analysis with previously measured $e^{-} p$ neutral current data. The measured cross-sections are compared to the predictions.
\end{abstract}

XXI International Workshop on Deep-Inelastic Scattering and Related Subjects 22-26 April, 2013

Marseilles, France

*Speaker. 


\section{Introduction}

The measurement of structure functions at HERA was one of the central goals of building the machine and the experiments. Structure functions provide a way of describing the internal structure of a proton and can be measured over a very wide range of squared four-momentum transfer, $Q^{2}$, and Bjorken $x$ at HERA. Such measurements are very interesting in their own right and also provide essential input to many other experiments, in particular as an important ingredient to cross-section calculations at hadron machines, such as the LHC.

Percent precision has been achieved over a wide kinematic range and, over the past several years, a further significant improvement in precision has been achieved by combining the ZEUS and H1 measurements [1]. The HERA II upgrade included the ability to provide longitudinally polarised $e^{-}$and $e^{+}$beams. Polarised beams allow electroweak effects to be observed and studied in $e p$ interactions.

The final ZEUS polarised $e^{-} p$ cross-sections have been published earlier [2]. This talk covers the final ZEUS publication on $e^{+} p$ scattering cross-sections at high $Q^{2}$ [3]. It is useful to express the measured cross-sections in terms of the so-called reduced cross-sections defined as:

$$
\tilde{\sigma}^{e^{ \pm} p}=\frac{x Q^{4}}{2 \pi \alpha^{2}} \frac{1}{Y_{+}} \frac{\mathrm{d} \sigma}{\mathrm{d} x \mathrm{~d} Q^{2}}=\tilde{F}_{2}\left(x, Q^{2}\right) \mp \frac{Y_{-}}{Y_{+}} x \tilde{F}_{3}\left(x, Q^{2}\right)-\frac{y^{2}}{Y_{+}} \tilde{F}_{L}\left(x, Q^{2}\right)
$$

where $\alpha$ is the fine-structure constant, $Y_{ \pm}=1 \pm(1-y)^{2}$ and $y$ is the inelasticity. $\tilde{F}_{2}\left(x, Q^{2}\right), \tilde{F}_{3}\left(x, Q^{2}\right)$ and $\tilde{F}_{L}\left(x, Q^{2}\right)$ are generalised structure functions.

This talk concentrates on looking for electroweak effects in the scattering cross-sections. This is done by either comparing the $e^{-} p$ and $e^{+} p$ cross-sections or making use of the longitudinal polarisation. Electroweak effects are quantified in the structure function $\tilde{F}_{3}$. It can be extracted for example from the difference of the reduced cross-sections:

$$
x \tilde{F}_{3}=\frac{Y_{+}}{2 Y_{-}}\left(\tilde{\sigma}^{e^{-} p}-\tilde{\sigma}^{e^{+} p}\right),
$$

For the unpolarised case and given that the vector coupling to the electron to the $Z$ is small, $x F_{3}$ can be interpreted as

$$
x \tilde{F}_{3} \simeq-a_{e} \chi_{z} x F_{3}^{\gamma Z},
$$

where $a_{e}$ is the axial coupling of the electron to the $Z$ boson, $a_{e}=-1 / 2$, and $\chi_{Z}=\frac{1}{\sin ^{2} 2 \theta_{W}} \frac{Q^{2}}{M_{Z}^{2}+Q^{2}}$ and the superscript $\gamma Z$ denotes the term arising from the interference between photon and $Z$ exchange. $M_{Z}$ is the mass of $Z$ boson and $\theta_{W}$ is the weak mixing angle. $x F_{3}^{\gamma Z}$ can in turn be expressed as the difference between the quark and antiquark parton density functions (PDF):

$$
x F_{3}^{\gamma Z}=\sum_{q} e_{q} a_{q} 2 x(q-\bar{q})
$$

Assuming that the sea quark distributions are symmetric we obtain

$$
x F_{3}^{\gamma Z}=2 x\left[e_{u} a_{u} u_{v}+e_{d} a_{d} d_{v}\right]=\frac{x}{3}\left(2 u_{v}+d_{v}\right),
$$

with $a_{u}=1 / 2$ and $a_{d}=-1 / 2, u_{v}$ and $d_{v}$ are the $u$ - and $d$-quark valence PDFs, respectively, and $e_{q}$ is the quark electric charge. 


\section{Event selection and data samples}

The $e^{+} p$ measurements are based on $(135.5 \pm 2.5) \mathrm{pb}^{-1}$ of data collected in 2006-2007, of which $(78.8 \pm 1.4) \mathrm{pb}^{-1}$ were collected at a luminosity-weighted lepton-beam polarisation of $P_{e}=$ $0.32 \pm 0.01$ and $56.7 \mathrm{pb}^{-1}$ at $P_{e}=-0.36 \pm 0.01$.

Monte Carlo signal samples were generated using HERACLES [4] and DJANGOH[5, 6] with the CTEQ5D PDF set [7]. The hadronic final state was simulated using the colour dipole model of ARIADNE 4.12 [8] and JETSET 7.4 [9-11] was used for the hadronisation.

Events are selected requiring a trigger on an electron or large transverse energy in the detector. Offline, the scattered electron must have energy $E_{e}^{\prime}>10 \mathrm{GeV}$. The kinematic requirements are $Q^{2}>185 \mathrm{GeV}^{2}$ and $y<0.9$, where $Q^{2}$ is determined using the scattering angles of the electron and the hadronic system (double-angle method, denoted with subscript DA). The event is required to have a well reconstructed vertex with the requirement $|Z|<30 \mathrm{~cm}$ and a cut on the polar angle of the hadronic system is made to minimise the effects of the hole for the beam-pipe in the calorimeter.

In order to reject background, further cuts are applied: $38<E-P_{Z}<65 \mathrm{GeV}$, $P_{T} / \sqrt{E_{T}}<4 \sqrt{\mathrm{GeV}}$ and $P_{T} / E_{T}<0.7$ where $P_{T}$ and $E_{T}$ are the net transverse momentum and energy, and $E$ and $P_{Z}$ are the total energy and the sum of the $Z$ components, calculated from $P_{Z}=\sum_{i} E_{i} \cos \theta_{i}$, where $\theta$ is the polar angle measured with respect to the interaction vertex. The sums run over all calorimeter energy deposits.

The data are compared to Monte Carlo (MC) samples in Fig. 1. Very good agreement is seen. Achieving the level of agreement between data and MC simulation that can be seen in these figures is a lot of work. Comparison with measurements made using the HERA I dataset show that HERA II has the advantage of a larger dataset and a better and more robust determination of the primary vertex. However, there is more material in the inner detector that requires a more detailed and accurate simulation of this material in the Monte Carlo.

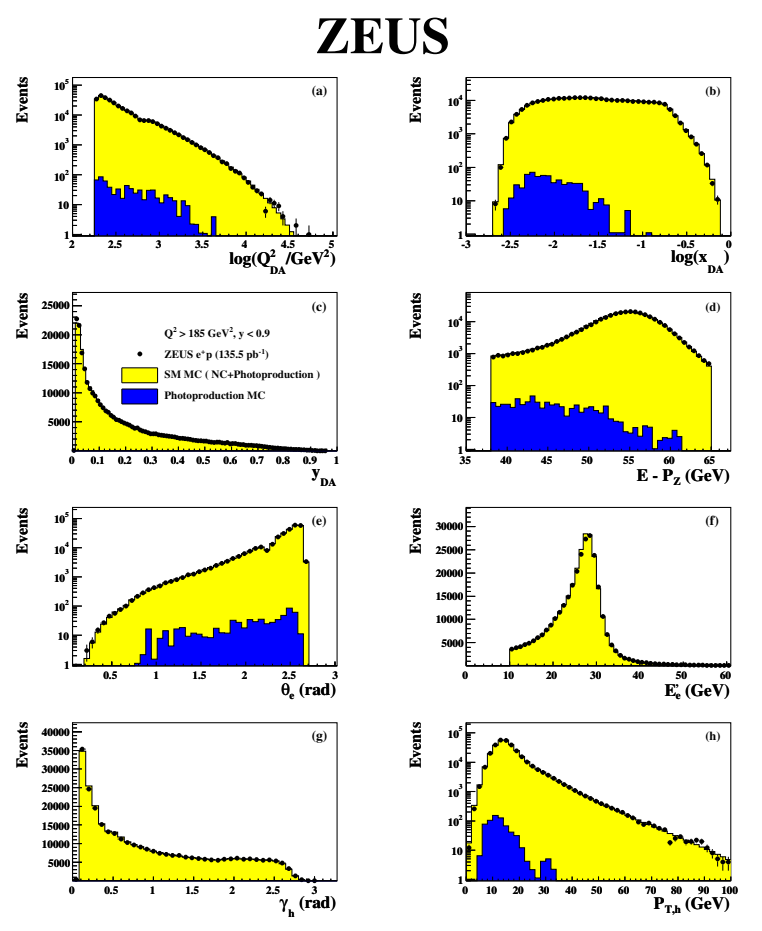

Figure 1: Comparison of the $e^{+} p$ NC data sample with predictions from the MC simulation. Distributions of (a) $Q^{2}$, (b) $x$, (c) $y$, (d) $E-P_{Z}$, (e) $\theta_{e}$ (f) $E_{e}^{\prime}$, (g) $\gamma_{h}$ and (h) $P_{T, h}$ are shown. The subscript $e$ indicates the scattered electron and $h$ the hadronic system.

\section{Results}

Using the whole $e^{+} p$ dataset, the measured cross-section as a function of $Q^{2}$ is shown in Fig. 2 
and compared to the Standard Model (SM) predictions with different PDFs. The kinematic range is defined as $Q^{2}>185 \mathrm{GeV}^{2}, y<0.9$ and $y(1-x)^{2}>0.004$. The residual polarisation in the data is 0.03 and this is corrected to $P_{e}=0$ using theory. The measurements are compared to predictions using several different PDFs. Note that the luminosity uncertainty (1.8-1.9\%) is not included in the error band. The predictions with different PDFs all agree well with the data.

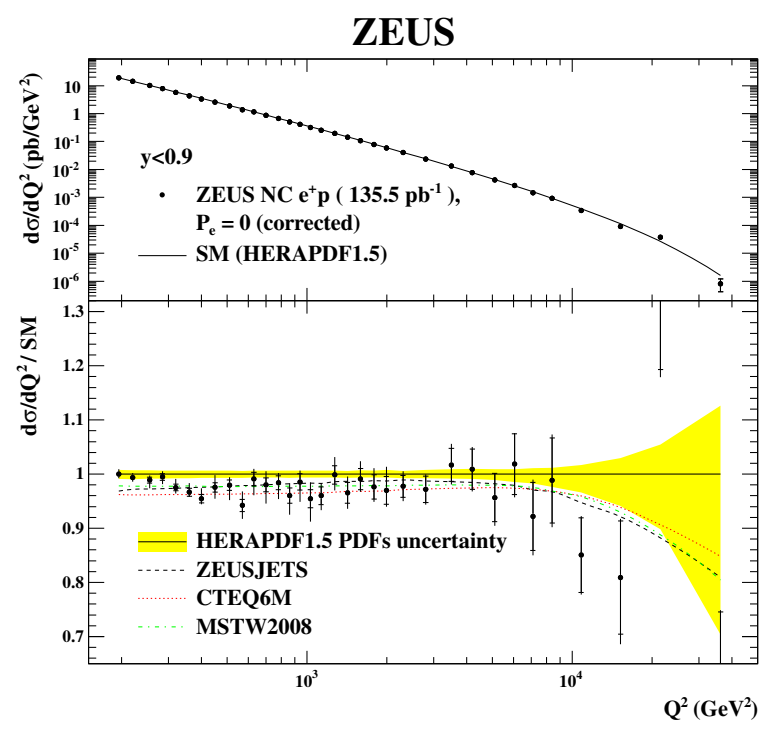

Figure 2: The measured differential cross-section $\mathrm{d} \sigma / \mathrm{d} Q^{2}$. The upper figure shows the cross-section compared to the SM prediction using the HERAPDF 1.5 PDF [12]. The lower figure shows the ratio of the data and different PDFs to the HERAPDF 1.5 prediction.

Figure 3 shows the cross-section as a function of $y$ for two different $Q^{2}$ cuts. In the left-hand plot the cross-section in dominated by photon exchange, while in the right-hand plot electroweak effects play a role. The precision of the measurements is clearly visible.

The cross-sections for negative and positively polarised positrons are compared in Fig. 4. The measurements with both polarisations are in good agreement with the predictions.

Figure 5 shows the ratio of the single differential cross-sections as a function of $Q^{2}$. A clear

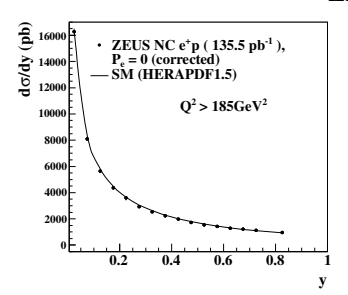

ZEUS

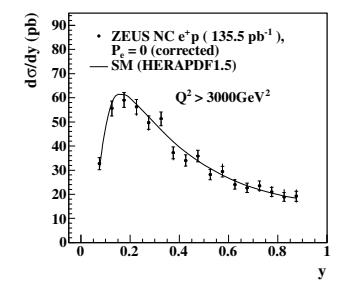

Figure 3: The measured differential crosssections $\mathrm{d} \sigma / \mathrm{d} y$ for two different $Q^{2}$ cuts. The data are compared to the SM prediction using HERAPDF 1.5. difference between the two polarisations is seen at high $Q^{2}$. The asymmetry $A^{+}$is defined as

$$
A^{+}=\frac{2}{P_{e,+}-P_{e,-}} \frac{\sigma^{+}\left(P_{e,+}\right)-\sigma^{+}\left(P_{e,-}\right)}{\sigma^{+}\left(P_{e,+}\right)+\sigma^{+}\left(P_{e,-}\right)}
$$

and shown in the lower figure, where $\sigma^{+}\left(P_{e,+}\right)$ and $\sigma^{+}\left(P_{e,-}\right)$ are the differential $e^{+} p$ cross-sections evaluated at positive and negative polarisation values $P_{e,+}$ and $P_{e,-}$. 


\section{ZEUS}
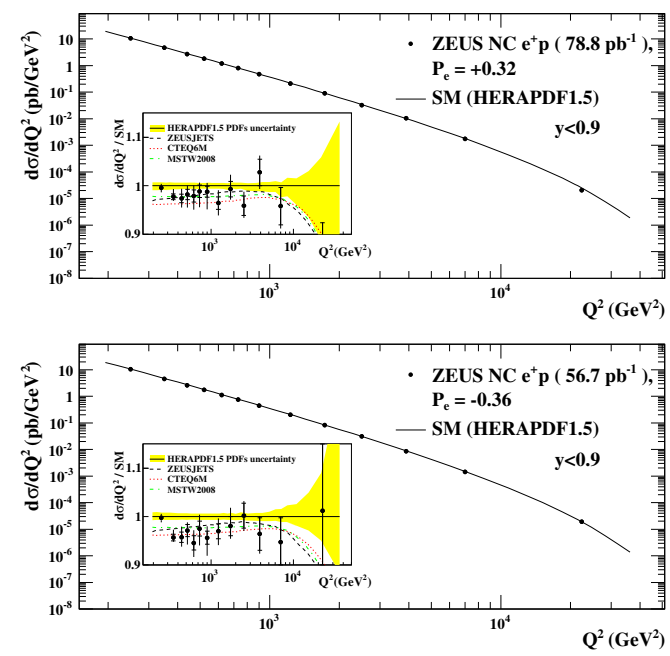

Figure 4: $\mathrm{d} \sigma / \mathrm{d} Q^{2}$ for positively (top) and negatively (bottom) polarised positrons compared to the SM prediction using HERAPDF 1.5. The inserts show the ratio of the measured and predicted cross-sections to the SM prediction using HERAPDF 1.5 .
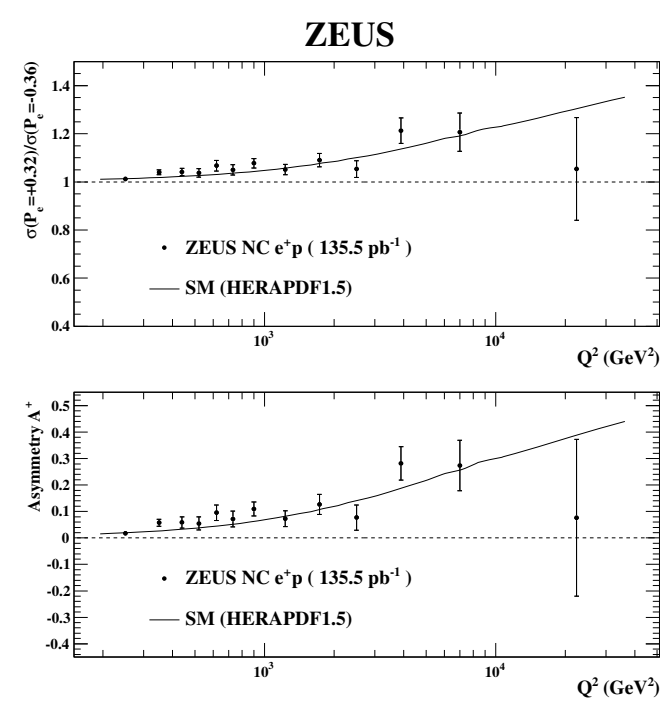

Figure 5: The ratio of $\mathrm{d} \sigma / \mathrm{d} Q^{2}$ for positive and negative polarisation (top) and the polarisation asymmetry $A_{+}$as a function of $Q^{2}$.

For equal and opposite polarisations and keeping only leading terms, $A^{+}$can be written as

$$
A^{+}=\simeq-\chi_{Z} a_{e} F_{2}^{\gamma Z} / F_{2}^{\gamma}=-2 \chi_{Z} a_{e} v_{q} / e_{q}
$$

To first order it is insensitive to the PDFs and gives direct evidence of parity violation with minimal assumptions on the proton structure.

\section{Summary}

The neutral current cross-section measurements at high $Q^{2}$ from ZEUS have now been completed and published. Comparing the cross-sections for positive and negative polarisation and also for $e^{-} p$ with $e^{+} p$ clear evidence for electroweak effects and parity violation is seen. These measurements provide a stringent test of electroweak effect in the Standard Model.

\section{References}

[1] H1 and ZEUS Collabs., F. Aaron et al., JHEP 01 (2010) 109, arXiv: 0911.0884 [hep-ex].

[2] ZEUS Collab., S. Chekanov et al., Eur. Phys. J. C 62 (2009) 625, DOI: $10.1140 /$ epjc/s10052-009-1055-6, arXiv: 0901.2385 [hep-ex].

[3] ZEUS Collab., H. Abramowicz et al., Phys. Rev. D 87 (2013) 052014, arXiv: 1208.6138 [hep-ex] . 
[4] A. Kwiatkowski, H. Spiesberger and H.-J. Möhring, Comp. Phys. Comm. 69 (1992), also in Proc. Workshop Physics at HERA, ed. by W. Buchmüller and G. Ingelman, (DESY, Hamburg), (1991) 155, DOI: $10.1016 / 0010-4655$ (92) 90136-M.

[5] G. Schuler and H. Spiesberger, Proc. Workshop on Physics at HERA, ed. by W. Buchmüller and G. Ingelman, vol. 3, (DESY, Hamburg), (1991) 1419.

[6] H. Spiesberger, (1998), URL: http://www. desy.de/ hspiesb/djangoh.html.

[7] CTEQ Collab., H. Lai et al., Eur. Phys. J. C 12 (2000) 375, DOI: $10.1007 / \mathrm{s} 100529900196$.

[8] L. Lönnblad, Comp. Phys. Comm. 71 (1992) 15, DOI: $10.1016 / 0010-4655$ (92) 90068-A.

[9] T. Sjöstrand, Comp. Phys. Comm. 39 (1986) 347, DOI: $10.1016 / 0010-4655$ (86) 90096-2.

[10] T. Sjöstrand and M. Bengtsson, Comp. Phys. Comm. 43 (1987) 367, DOI: $10.1016 / 0010-4655$ (87) 90054-3.

[11] T. Sjöstrand, Comp. Phys. Comm. 82 (1994) 74, DOI: $10.1016 / 0010-4655$ (94) 90132-5.

[12] H1 and ZEUS Collabs., H. Abramowicz et al., H1prelim-10-042, ZEUS-prel-10-018, 2010, URL: https://www.desy.de/hl zeus/combined_results/herapdftable. 